SHS Web of Conferences 24, 02009 (2016)

DOI: $10.1051 /$ shsconf/20162402009

(c) Owned by the authors, published by EDP Sciences, 2016

\title{
Exploration and analysis of rural primary school teacher's language violence
}

\author{
Honglian Deng ${ }^{1} \&$ Zhaoxia Lei $^{2}$ \\ ${ }^{1}$ School of Education, Hubei University of Science and Technology, Xianning, Hubei, China \\ ${ }^{2}$ Jiayu Experimental Primary School, Xianning, Hubei, China
}

\begin{abstract}
As the problem of rural education becomes more and more obvious while the supervision on stay-at-home children's education becomes more and more difficult, rural primary school teacher's language violence has become a new big problem today. This paper collected and investigated the improper language used by rural primary school teachers so as to analyze the features, harm, causes and solutions of language violence, trying to explore and analyze rural primary school teacher's language violence from perspective of sociology and remind primary school teachers of rethinking. In subjective aspect, this paper hopes to improve rural primary school teacher's comprehensive quality, establish specification for teacher's language, lower rural teacher's vocational burnout and alleviate the psychological pressure that exam-oriented education and rural stay-at-home children impose on teachers. In objective aspect, this paper hopes to enhance the supervision from society and administrative departments for education. All the above measures can be taken to effectively eliminate teacher's language violence and resolve the crisis.
\end{abstract}

Keywords: primary school; teacher; language violence

\section{INTRODUCTION}

Language is an important tool for teachers to preach their knowledge and clear up solutions to their students. There is certain specialty contained in primary school teacher's education and teaching language that plays an important role in children's development. Primary school teacher's language charm can erect a bridge to build mutual communication with primary school students, helping make complicated things simple, abstract things concrete and plain things amazing in order to arouse student's interest in learning and lead them the way into ocean of knowledge. On the contrary, they may leave many serious negative influences or even huge harm on primary school students if primary school teachers use language violence in education teaching. New word "language violence" is popular today. It has obtained great attention from educators. According to report, language violence is perplexing children at school.

\section{RESEARCH BACKGROUND}

Where there is violence, there is harm, either physical or psychological. On the face of it, language violence looks harmless to us as it would only cause huge psychological pain in our mood, self-esteem and heart. In fact, it is not. The spiritual harm that language violence leaves on us can be much worse than physical pain. The worst result is that language violence can put human life in danger. Take Xianglinsao in $\mathrm{Lu}$ Xun's novel "Blessing" as an example. In the end, Xianglinsao's fate changed because of Si Shen's "Xianglinsao, you can just leave it alone". She felt desperate by this extremely cold-hearted sentence and got her mind somewhat unhinged. At last, Xianglinsao could not work anymore and had to go begging. Finally, she died in snow, sadly.

Primary school teachers should be the guides of young children. They should speak in a gentle and mild way to educate students in their education and teaching activities. However, nobody's perfect and teachers are not sages or men of virtue. In fact, they may use improper words in a moment of anger while educating students. This kind of language violence can harm on student's self-esteem, enhance student's inferiority complex and completely destroy student's confidence. Relation between teachers and students may become extremely tense and make students feel anx- 
ious and depressed. In this situation, students may develop a psychological habit of avoiding and running away which can bring emotional disorder, mental abnormal symptoms, and even loss of courage to live.

For example, on August $31^{\text {st }}, 2003$, Chutian Metropolis Daily reported a real event happened in a primary school of Hankou, Wuhan with title of "A Nickname of 'Locust' can Hurt, Teacher Made Trouble by Giving Student Nickname": a substitution math teacher called a student "locust" ("Huangchong" in Chinese) many times in the class because of the student's family name and hurt the student's self-esteem seriously. As a result, the student's rating in class dropped from the middle level to the lowest and he had to be transferred to another school. In September, 2004, Cao Tenan who was in the third grade of a primary school ate an apple in the class. The teacher shouted at him and told him to "get out". Cao Tenan suffered from great mental harm because of the teacher's shouting and the classmates' laughter. According to judicial expertise, Cao Tenan got "Retardance Response" which is a kind of mental disorder. According to media coverage, a 14-year-old boy in Inner Mongolia hung himself and died, because he was scolded by the teacher as "Scumbag, you scumbag" after throwing ink on the teacher's back.

From the above, it can be concluded that language violence can affect primary school student's interest in study, insult children's personality, hurt their self-esteem and confidence and may even seriously hurt their personality development. In some situations, children may form "disintegrated personality" or "aggressive personality". Some may even lose the courage to live due to the lack of self-esteem. Nowadays, language violence has become a new big problem of education in China. Meanwhile, it is also a social problem that is easily to be neglected.

\section{LITERATURE REVIEW}

Foreign scholars mainly conduct predictability study of teacher's language violence from perspective of operational definition, such as the nomination research method used to study teacher's language violence. There are not many studies of teacher's language violence; most of them are a small number of status surveys and longitudinal predictability research represented by Brendgen.

Domestic researchers conduct their study mainly from the aspects of teacher's language violence types, causes, and countermeasures. Some researchers conduct study of the negative effect that teacher's language violence leaves on students from perspective of psychology. For example, Li-Fang Shen and Ying Zhu divided language violence into contempt type, sarcasm type, irony type, consideration type, beating type, vertical and horizontal type, sickness type, framing-up type, stand-in-the-corner type and threat type to study in their article names Status Analysis and Revising Strategies of Middle/Primary School Teacher's Language Violence. In his Causes and Countermeasures of Campus Language Violence, Hua-An Chen summarized the causes of campus language violence in two aspects: causes on teacher and causes on student. Causes on teacher include the followings: the low level and lack of teacher's comprehensive quality and humanistic spirit, the problems in the elitism value of our educational system, the problems existing in teacher's psychological health and the problems of the educational management and assessment implemented by schools. In their Rational Thinking on Teacher's Language Violence, Ai-Yun Zhang and Gui-Lan Nie pointed out that language violence can reflect the problems existing in our school education. In his $I n-$ vestigation Research on Middle/High School Student's Language Violence, Li-Zeng Zhang macroscopically analyzed the threat and negative effect that continuously emerging language violence which is caused by society, parents, schools and teachers has on middle/high school and primary school students. Xue-Mei Zhang offered some countermeasures and suggestions to deal with teacher's language violence in her Investigation Report on Teacher's Language Violence: (1) Improve social awareness of language violence problem; (2) perfect corresponding laws and regulations; (3) establish effective mechanism for complaints and supervision. From all the above, it can be seen that the study and theory of teacher's language violence are in development while related theories and research of domestic and foreign studies are increasing. They all have provided important reference to my study. I will try to explore and analyze the features, causes and solutions of rural primary school teacher's language violence from perspective of sociology.

\section{FORM OF LANGUAGE VIOLENCE}

From July to December, 2005, Beijing Teenager's Legal Aid and Research Center conducted questionnaire inquiry to 315 primary/middle/high school students from 30 schools and released the "Investigation Report on Teacher's Language Violence". According to statistics, $51 \%$ of the primary school students, $72 \%$ of the middle school students and $9 \%$ of the high school students think teacher's language violence has caused mental harm to them. Primary school students think teacher's language violence make them "become scared of teachers" and "get self-esteem hurt" [1]

For what is teacher's language violence, the understanding is simple in most cases. Generally, teacher's language violence refers to the criticism, scolding and cynicism that teachers use while criticizing and educating their students. In fact, teacher's language violence is far beyond this definition, either in form or in content. In our opinion, the so-called teacher's violence refers to teacher's verbal behaviors which cause 
physical harm to student's mental health during educational teaching activities. In communication with primary/middle/high school teachers, the main forms of language violence can be summarized as the followings:

Isolation - "Boys and girls, what's his behavior to be implied?" "He has ruined this class. What do you think we should do?"

Sarcasm - "How can you expect yourself to learn piano, you stupid head?" "How did you grow up by being so stupid!"

Comparison - "You can never be compared with John Doe. There is a milky way between you two."

Complain - "I cannot enlighten you anymore. Call your dad to come here." "Come, let's go to the President's Office."

Prediction - "I bet you will get into jail one day."

"You stupid. You can never learn anything."

Conclusion - "You are the unique freak in the whole school." "Is there anyone more stupid than you in the world?"

Keep-in-record - "Remember it. I'll deal with you sooner or later!" "See you in your graduation appraisal."

Abusing — "You block-headed!" "Are your parents in intermarriage?"

Irony - "You've got a lot of nerve. If I were you, I would have found a crack on the ground to hide." "Look at you! You don't look like my student at all."

Threat - "If you dare to do it again, I'll make you remember me forever." "If you talk again, I'll stop teaching. You can get another teacher."

Berating - "I have taught for so many years, but I've never seen anyone like you!" ${ }^{[2]}$..

\section{CAUSES OF RURAL PRIMARY SCHOOL TEACHER'S LANGUAGE VIOLENCE}

\subsection{Causes on teacher}

The phenomenon that language violence has always existed in school education can be explained by the problems in teacher's comprehensive quality and humanistic spirit.

First of all, in modern school education, one of the main reasons for why some rural primary school teachers impose language violence on their students is because they are lack of strong pedagogical knowledge and psychological knowledge. There's no new-type relation that is equal, cooperative and conversationally interactive between teachers and students. Current relations between teachers and students are still principle-and-subordinate type in which teachers are in powerful position while students are in weak position. Some teachers use simple and rude education methods because their education concepts are backward; they may even give physical punishment to students sometimes. Thus, it is not hard to understand why teachers use improper language to students who talk, take petty actions, and fail to finish homework, get bad scores in exams and always make mistakes in the class. The emerging language violence in education and teaching can well reflect the fact that teacher's comprehensive quality cannot meet the requirements set in quality education. Evaluation Recommendations of Implementation Proposals in the third part of Chinese Curriculum Standards for Primary Schools (2011) clearly point out that: Teacher's assessment shall fully respect student's individual differences so as to guarantee that each student can have healthy development.

Second, another main reason is that primary school teachers have low income but great working pressure. The current society is in rapid transformation period with continuous economic development. Primary school teachers do not have high income and have to support their family. Due to regional factors, the salary that primary school teachers in rural areas can get is even lower and thus cause serious imbalance in those teacher's heart. Teachers are facing great vocational competition, high requirements in education background and professional title, great pressure from lowest place elimination system and higher requirements set in new curriculum reform. In this new era, it is hard to teach students and difficult to be a teacher with low income and hard work. In some schools, teacher's employment and performance pay are closely related to student's exam scores after the implementation of new curriculum reform. Teachers are facing higher expectations from society, schools and parents. It is natural that all these can bring teachers negative mood and lack of patience in education. Therefore, the existence of language violence in education and teaching activities is not surprising. However, language violence can not only block emotional communication between teachers and students objectively, but also create inharmonious atmosphere in emotion between them subjectively and directly impact education and teaching effect.

Moreover, primary school teacher's vocational burnout is an important factor in language violence. Many primary school teachers have worked in the same position for more than two decades. They have generated vocational burnout long ago and lost their patience and enthusiasm on work and students. When they face complicated situation or naughty students, it is normal for them to use language violence. Under "human-oriented" ideological guidance, if language violence can be eliminated to create harmonious atmosphere in emotion between teachers and students, teachers can keep their enthusiasm on teaching students while students can understand the care and hardship of their teachers. By this means, students can have their confidence in study enhanced and their limitless interest in study aroused. In this situation, teachers and students can build up close relations and reach the highest level of teaching and learning. As a 


\section{SHS Web of Conferences}

result, students are able to develop healthy personality.

In addition, many primary school teachers think primary school students are too young to care about what they say. In their mind, middle school or high school students are in rebellion period with tall and strong physical shapes, so they need to think carefully before saying anything to these students, and college students are adults who need to be respected in every aspect. Unlike middle/high school students or college students, those teachers think primary school students will not remember the harsh words they use. Therefore, they seldom think twice and carefully choose the words they use while criticizing and educating their students.

\subsection{Causes on student}

Most primary school students in rural areas are stay-at-home children who are easy to develop bad behavioral habits and poor learning habits. With the addition that children in primary school are fond of play due to their nature, it's hard for them to obey the rule in the class. They always talk and have petty actions; some even eat in the class. After class, they always make noise by playing and chasing classmates. Once a teacher sees any of these phenomena, he would stop it and criticize the students. If he uses an improper method, language violence may occur. As there are differences between the management of primary school curriculum and that of kindergarten, primary school students in lower grades need a long time to adapt to the change from kindergarten to primary school. During this period, students' study goals may not be clear and their internal learning drive may be weak. They may not have the right study attitude and proper behavior in the class. With the psychological effect of exasperating at student's failure to make good, teachers will criticize and educate students in a harsh way. If the language they use is improper or out of normal range, the language violence generated herein can hurt primary school student's heart and spirit.

\subsection{Lack of supervision from society and education management administrations}

With the official implementation of The Law on the Protection of Minors, administrative departments for education and schools start to pay more attention to the supervision on physical punishment. Violent actions to give students physical punishment are basically not existent anymore. However, some teachers still have language violence and no sufficient attention is attached to them by administrative departments for education, schools and parents. Many children hold the depression and shame generated by their teacher's scolding and criticism in heart and seldom tell their parents. As a result, the harm that students suffer from this phenomenon is concealed, leading to the lack of supervision on teacher's language violence. Hence, language violence still prevails in primary schools.

\section{APPROACHES TO ELIMINATE LANGUAGE VIOLENCE}

\subsection{Improve primary school teacher's vocational quality}

\subsubsection{Study and update of pedagogical and psychological knowledge}

"Language violence" comes from mouth but originates from "heart". It can reflect the lagging situation of teacher's professional development which requires lifetime learning, especially continuous learning of pedagogical and psychological knowledge, so as to solve constantly emerging new problems and catch up with the pace of student's development ${ }^{[4]}$. For example, if a kid makes a mistake at school, a teacher may use a simple method to deal with it by saying "ask your parent(s) to come tomorrow". This sentence sounds quite simple and harmless to student. However, it is not hard to understand how the kid will be scared of asking his/her parent(s) to come to school. After hearing this, the kid will not rethink what mistake he/she has made or how to correct it. Instead, most of his/her attention will be paid to feel anxious and nervous, thinking about how to explain to his/her parent(s). Therefore, the request of asking parents to come to school is not the best way for education. In fact, teachers should insist on the idea of "all for student's development", enhance the concept of "human-oriented", get along with students and treat students with all sincerity. Teachers should make use of all means to study new knowledge of pedagogy and psychology. Besides, they should also pay attention to the features of student's development, care more about student's merits in education teaching and try to use the charm of language to encourage their students.

\subsubsection{Enhancement on teacher's professional happiness}

Although rural primary school teachers are suffering from low income, great working pressure and increasing vocational burnout, they should still try to find pleasure from hardship and realize the value of being a teacher. Primary school teachers get along with innocent and lively children. In their students' eyes, they are sacred and great. To keep the childlike innocence of their students, they should continuously study hard so as to improve their quality and teacher's ethics, try to eliminate vocational burnout and enhance their own vocational happiness. No language violence shall be imposed on primary school students.

\subsubsection{Improvement on language quality}

Teacher's verbal language sounds simple; but in fact, 
it is not. The use of verbal language is extremely important in teaching and cultivation. Different methods, tones, intonations and sentence patterns to express the same meaning can bring different experience to listeners. For example, a kid did a great job in one exam. His teacher was supposed to praise and encourage him. However, what he said was "Boys and girls, pay attention to see that (an underachiever like) John Doe can also get a high score". After hearing this sentence, the class burst into laughter and John Doe's face turned crimson with anger and shame. What the teacher said did not only fail to receive the expected effect, but also break his student's self-esteem. The teacher didn't realize that his way of speaking can destroy an underachiever's self-esteem completely. Actually, if the teacher could change his way of speaking and said "John Doe has made great progress these days. If you have the right attitude and study in the right way, you can also improve your learning efficiency and get a high score like John Doe". This sentence can praise John Doe and tell the other students to develop the right attitude and pay attention to their way of study at the same time. This kind of teacher's language can be called the language full of charm and without violence. Therefore, teachers should have right understanding of the harm that language violence can cause, pay attention to improve their language quality while improving their own quality and culture and study the art of education language.

\subsection{Improve the function of family education}

In children's education, family function is far more than school function while parent's function is far more than teacher's. High-quality parents have stronger sense to understand this fact. However, the major part of parents have little awareness of it, especially parents come from rural areas and work in urban areas. In their mind, culture and knowledge are exactly what children study at school, thus it is teacher's responsibility and obligation to teach their children how to do things and how to be a man. This kind of parents transfers the responsibility of family education to school completely. Due to lack of family education, students may have more problems in study, personality, psychology and habit. In addition, as most primary schools apply large-class quota, a classroom may hold 50 to 60 students or even 70 to 80 students sometimes. If a student's problem cannot be solved at school, it has to be solved at home. After family function is weakened, teachers have to bear greater workload. As a result, they may inevitably use improper language while criticizing and educating students. If every parent can improve the function of family and stop shirking family education responsibility, many problems that students have can be solved at home. In turn, students can behave better at school. In this way, relation between teachers and students can be pleasant and harmonious with much less language violence.

\subsection{Enhance the supervision of each aspect}

After the implementation of The Law on the Protection of Minors, there is much less physical punishment on students which is delightful. However, there's still lack of enough attention paid to the harm of teacher's language violence by students, parents, teachers and administrative departments in charge of education. Teacher's language violence can still be found at school today. Administrative departments in charge of education are lack of enough awareness of the harm that can be caused by language violence while parents always neglect it. It is a pathetic fact. From the above, it can be seen that the supervision of every aspect must be enhanced to eliminate primary school teacher's language violence. Administrative departments in charge of education should emphasize on the construction of professional ethics. They can expressly stipulate that no teacher is allowed to give corporal punishment on students in any form and no language violence shall be made. And they can also establish a request mechanism for students and parents to complain about teacher's language violence. Parents should give more care to their children. Primary school students always show their happiness or sadness on their face, but few of them would ever tell their parents when they suffer from teacher's language violence at school. Therefore, parents should pay attention to their children's mood every day. Once they find their children feel down, they should ask and find out why. If the children are actually scolded or criticized any teacher, parents should communicate with the teacher. Students and parents should dare to say no to teacher's language violence. All these measures can supervise teacher's actions in a subtle way and restrain teacher's language violence to a certain extent.

"Whoever wins education can win the $21^{\text {st }}$ century." Primary school students are the future of our country, and primary school teachers are students' guides and designers. Primary school teacher's language violence can leave serious negative influence on student's personality development, physical and psychological health, and social communication. As a result, we should try to solve teacher's problems of psychological health and vocational burnout, improve their comprehensive quality and humanistic spirit and encourage them to use proper education language and wisdom to teach their students. Moreover, we should pay special attention not to be "tough in mouth" to primary school students and give more positive assessment to them. We hope that teacher's language can be full of charm and without violence.

\section{ACKNOWLEDGEMENT}

This paper is financially supported by the research 
project of Hubei University of Science and Technology - Linguistic Research of Primary School Teachers (kt12002).

\section{REFERENCES}

[1] Zhang, X.M. 2006. Investigation report on teacher's language violence. Teacher's Journal, (06): 51-54.

[2] Liu, X.C. 2005. Schools refuse verbal abuse. Beijing Education, (05): 42-43.

[3] Liu, X.F. 2007. Analysis and Countermeasure Study of Primary School Teacher's Language Violence. Master's Thesis from Minzu University of China, pp.7-17.

[4] Mou, Y.X. 2006. Discussion on improper teacher's language from perspective of class culture. Journal of Chinese Education, (07): 76-78. 